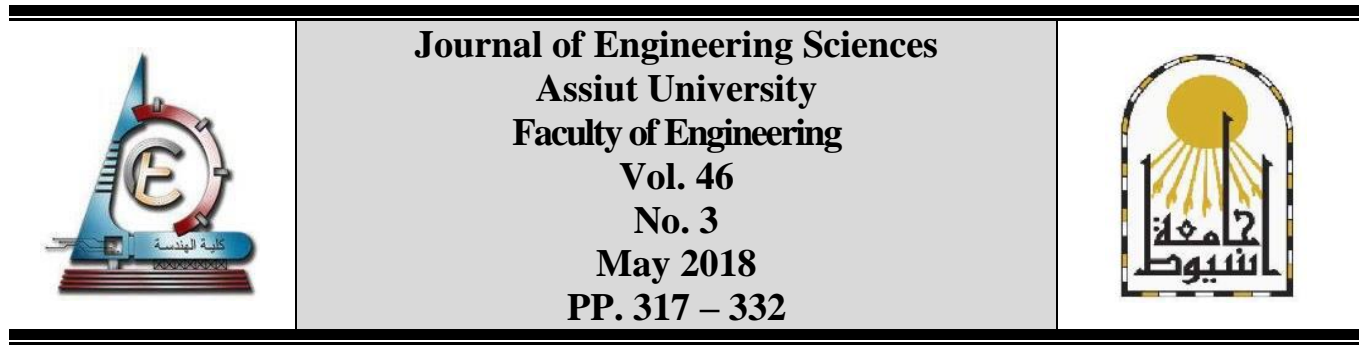

\title{
FAIR- M-ESTIMATOR BASED MIMO DETECTOR AND MIMO SYSTEM INVESTIGATION IN NON-GAUSSIAN CHANNELS
}

\section{Mohamed H. Essai}

Electrical Engineering Department, Faculty of Engineering-Qena, Al-Azhar University, Egypt. E-mail address: mhessai@azhar.edu.eg

Received 4 February 2018; Accepted 28 February 2018

\begin{abstract}
The impact of the presence of non-Gaussian noise on the efficiency of OSIC-MMSE and OSIC-ZF detectors for 2x2 SM-MIMO communication systems were investigated. Also, I investigated to what extent, increasing the number of transmitting and receiving antennas in SM-MIMO systems will enhance the efficiency of the investigated detectors against these non-Gaussian noises. Finally, a new M-Estimator based SM-MIMO detector named Fair detector is proposed, in order to achieve robust detection, for non-Gaussian channels. The proposed detector designed for LTE and LTE-advanced wireless communication systems. The proposed detector was compared with aforementioned detectors in terms of bit error rate, and computational complexity. Simulation results show the substantial performance of the proposed detector compared to the investigated detectors.
\end{abstract}

Keywords: Fair detector, Robust detector, SM-MIMO, uncertain channel noise model.

\section{Introduction}

As the frequency spectrum is considered as the most valuable resource for wireless communication systems, techniques are required to use the available bandwidth more effectively. MIMO technology is one of these techniques. A linear increase in the transmission data rates can be achieved using MIMO technologies which utilizing spatial multiplexing transmission technique (SM). Through SM-MIMO system, independent data sub-streams can be transmitted effectively within the system's operating bandwidth. SM permits to every data sub-stream to handle with the same channel characteristics that would be handled by the SISO systems. The SM-MIMO system improves the capacity of the communication system by a multiplicative coefficient equals to the number of independent sub-streams of the transmitted data [1], [2].

The most recent standards in the sphere of wireless communication such as LTE and LTE-advanced use SM-MIMO in order to grant high data rates at high speeds while retaining the predefined QoS. LTE provides $300 \mathrm{Mbps}$ at Down-Link (DL), and $75 \mathrm{Mbps}$ at Up-Link (UL), while LTE-advanced provides 1Gbps at DL, $500 \mathrm{Mbps}$ at UL. These supported data rates forced by the MIMO configuration and users' demands [3]. 
The design of MIMO detector that separates independent data sub-streams at the receiving end is the key component of MIMO systems in terms of performance improvement and reasonable complexity. The optimum MIMO detectors were developed for wireless communication systems with additive white Gaussian noise (AWGN). Optimum detectors are usually not suitable for practical implementation due to their high computational complexity.

In the course of overcoming the difficulties of the practical realization of optimum detectors, suboptimum detectors have been well-developed for communication systems with AWGN. Suboptimum detectors have a low computational cost, which makes it is possible to implement them in practice, but their performance is generally less than that of optimum detectors.

The suboptimum -based SM-MIMO detectors include Zero Forcing (ZF), Minimum Mean Square Error (MMSE) detectors, and Ordered Sequence Interference Cancelation detectors that may base on the ZF (ZF-OSIC) or base on MMSE (MMSE-OSIC). All detectors have a significant task that is to decrease the bit error probability.

There are continuous efforts in order to build up detectors that achieving a semi-optimal or optimal performance with minimal complexity, in other words, reduces the performance shortage of the optimal detectors in comparison with the suboptimal detectors, in terms of the bit error probability.

In this paper, the robustness of OSIC-MMSE and OSIC-ZF detectors were examined for 2x2 SM-MIMO, in order to explore its performance under the conditions of Gaussian and non-Gaussian channels. It was noted that the performances of the examined detectors degrade at heavy-tailed noises.

Also, the robustness of examined detectors was explored for 4x4 and 8x8 SM-MIMO systems, because of investigating the role of increasing the number of transmitting and receiving antennas in MIMO systems on the process of alleviating the bad effect of the heavy-tailed noises on the performance of the examined detectors. It was noted that increasing the size of MIMO systems, enhances the performances of examined detectors.

In the course of synthesizing more robust MIMO detectors, I propose Fair-based SMMIMO detector. The proposed detector utilizes Fair criterion, which is one of the robust statistics M-estimators. The proposed detector is compared with the aforementioned detectors at various MIMO configurations (2x2, 4x4, and 8x8), also under the conditions of Gaussian and non-Gaussian channels (uncertain noise environments), were strictly synthesized for the impractical GN distribution hypothesis $[4,5,6]$.

The SM-MIMO systems can be classified into open loop and closed loop SM. In the OLSM, the channel matrix is not feed backed, while in the CLSM the optimum channel matrix is feed backed by the end user's apparatus to the transmitting end. In this paper, an OLSM approach on the LTE-downlink was taken into consideration.

This paper is organized as follows; Section 2 explains the MIMO systems and Generalized GN Distribution as a kind of a practical noise distribution. In Section 3 suboptimal detectors are explained. Section 4 elaborates the proposed Fair detector. In section 5 simulation results are introduced to verify the performance improvements. Conclusions are given in Section 6. 
Mohamed H. Essai, fair-M-estimator based mimo detector and mimo system investigation ....

\section{Description of mimo system}

Fig. 1 illustrates a MIMO system, which is constructed of $\left(N_{R} x N_{T}\right)$ antennas, where $N_{R}$ and $N_{T}$ are the number of receiving and transmitting antennas respectively. Each transmitting antenna transmits a different data sub-stream, while each receiving antenna may receive all transmitted data sub-streams from all transmitting antennas.

The channel for a specific delay can thus be described by what is known by the channel matrix. Let $\mathbf{H}$ denotes a channel matrix with $(j, i)$ th entry $h_{j i}$ for the channel gain between the $i$ th transmitting antenna and the $j$ th receiving antenna, $j=1,2, \ldots \ldots, N_{R}$, and $i=1,2, \ldots . ., N_{T}$. The coefficients of $\mathbf{H}$ describe all possible paths that data streams from different transmitting antennas may experience [7-9].

The spatially multiplexed user's data at the transmitting end, and the corresponding signals at the receiving end are represented by $\mathbf{x}=\left[x_{1}, x_{2}, \ldots ., x_{N_{T}}\right]^{T}$ and $\mathbf{y}=\left[y_{1}, y_{2}, \ldots . ., y_{N_{R}}\right]^{T}$ respectively, where $x_{i}$, and $y_{j}$ denote the transmitted signal from the $i$ th transmitting antenna and the received signal at the $j$ th receiving antenna, respectively. Let $Z_{j}$ denotes the WGN Gaussian noise with a variance of $\sigma_{Z}^{2}$ at the $j$ th receiving antenna and $\mathbf{h}_{i}$ denotes the $i$ th column vector of $\mathbf{H}$. Now, the $\operatorname{MIMO}\left(N_{R} x N_{T}\right)$ system is represented by

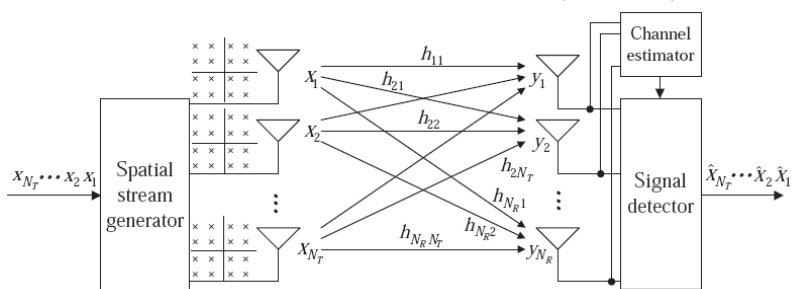

Fig. 1. SM-MIMO systems.

$$
\mathbf{y}=\mathbf{H x}+\mathbf{Z}=\mathbf{h}_{1} x_{1}+\mathbf{h}_{2} x_{2}+\ldots .+\mathbf{h}_{N_{T}} x_{N_{T}}+\mathbf{z}
$$

where $\mathbf{Z}=\left[Z_{1}, Z_{2}, \ldots . ., Z_{N_{R}}\right]^{T}[7-9],[18]$.

In [10] and [11], it was illustrated that an impulsive noise model is a reasonably actual description for numerous of communication channels, including metropolitan and indoor wireless environments. Impulsive noise can be characterized by heavy-tailed probability distributions and can be described by a number of statistical probability distributions.

In order to evaluate the efficiency of examined algorithms, it was evaluated by using computer simulation according to the probability of demodulation error per 1 bit (BER) versus SNR, at different noise distributions. In terms of the realistic noise distributions, the Generalized Normal (Gaussian) distribution (GG) PDF has been used as shown in equation (2).

$$
Z_{G G}(t, \alpha)=\frac{\alpha}{2 \sqrt{\Gamma(1 / \alpha) \Gamma(3 / \alpha)} \Gamma(1 / \alpha)} \exp \left\{-\left[\frac{|t|}{\sqrt{\Gamma(1 / \alpha) \Gamma(3 / \alpha)}}\right]^{\alpha}\right\}
$$


With different values of a shape parameter $\alpha$, where $\Gamma(t)=\int_{0}^{\infty} x^{t-1} e^{-x} d x$ is known as gamma function. The distribution in equation (2) is a priori unknown for all investigated detectors. GG-PDF in equation (2) has finite Fisher information and a variance equals to 1 for all $\alpha>0.5$. For $\alpha=2$, GG distribution coincides with the Normal distribution, while for $\alpha=1$, the distribution coincides with the two sided Laplace distribution. For $\alpha<2$, this distribution has heavier tails in comparison with the Normal distribution [8, 9, 18]. Fig.2 shows a variety of densities at different values of $\alpha$.

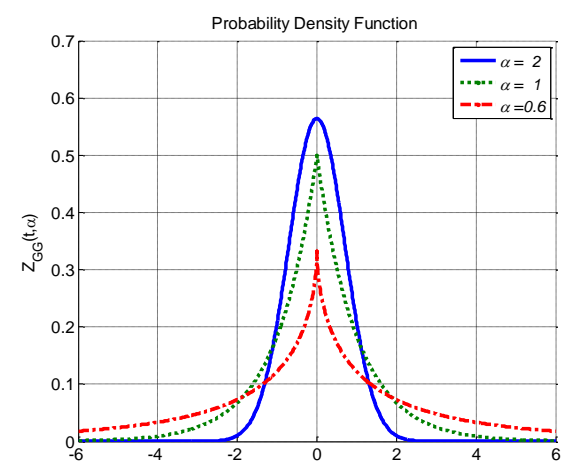

Fig. 2. Variety of noise distributions obtained by GG-PDF, at $\alpha=2,1$, and 0.6 .

\section{Suboptimum detectors}

\subsection{Zero-forcing detector}

The main task of the MIMO linear detectors is to estimate each of the multiple transmitted symbols by performing some of the linear operations to the observed samples at the receiving antennas. The major characteristic of the ZF detector is that it estimates many of estimations for each transmitted symbol which do not contain interference from the other transmitted symbols. ZF detector is commonly known as Decorrelator detector [12]. ZF detector aims to nullify the interference by using the following matrix:

$$
\mathbf{W}_{Z F}=\left(\mathbf{H}^{H} \mathbf{H}\right)^{-1} \mathbf{H}^{H}
$$

where $(.)^{H}$ is the Hermitian transpose operation. It inverts the channel effect as

$$
\begin{aligned}
x \mathscr{Z}_{F}=\mathbf{W}_{Z F} \mathbf{y} & =\mathbf{x}+\left(\mathbf{H}^{H} \mathbf{H}\right)^{-1} \mathbf{H}^{H} \mathbf{Z} \\
= & x+\mathbf{Z}_{Z F}^{o}
\end{aligned}
$$

Where $\mathbf{Z}_{Z F}^{o}=\mathbf{W}_{Z F} \mathbf{Z}=\left(\mathbf{H}^{H} \mathbf{H}\right)^{-1} \mathbf{H}^{H} \mathbf{Z}$. Note that the error of performance is directly related to the power of $\mathbf{z}_{Z F}^{o}$ [7-9], [18].

\subsection{Minimum mean square error detector}

Utilizing the SM-MIMO techniques, wireless communication systems that support high transmission data rates, and provide minimum errors can be implemented. SM-MIMO wireless communication systems that use the MMSE detector as a practical detector can achieve low computational complexity and support higher data rates. By using MMSE 
Mohamed H. Essai, fair-M-estimator based mimo detector and mimo system investigation ....

detector the desired signal energy would be strengthened, and the unwanted interferences would be nullified by maximizing its signal to interference plus noise (SINR) ratio. By using a MMSE detector, the wireless communication systems' capacities were shown to be scaled linearly with the number of the receiving antennas [3], [13-15]. The post-detection SINR can be maximized by using the MMSE criteria too. The used MMSE weight matrix is given as

$$
\mathbf{W}_{M M S E}=\left(\mathbf{H}^{H} \mathbf{H}+\sigma_{Z}^{2} \mathbf{I}\right)^{-1} \mathbf{H}^{H}
$$

For efficient MMSE detector performance, the noise statistical information of $\sigma^{2}$ is desired. Note that the $i$ th row vector $\mathbf{w}_{i, M M S E}$ of the weight matrix in equation (5) is offered by solving the next optimization equation:

$$
\mathbf{w}_{i, M M S E}=\underset{\mathbf{w}=\left(w_{1}, w_{2}, \ldots, w_{N_{T}}\right)}{\arg \max } \frac{\left|\mathbf{w h}_{i}\right|^{2} \mathrm{E}_{x}}{\mathrm{E}_{x} \sum_{j=1, j \neq i}^{N_{T}}|\mathbf{w h}|^{2}+\|\mathbf{w}\|^{2} \sigma_{Z}^{2}}
$$

Using the weight of MMSE in equation (5), we could obtain the next relationship:

$$
\begin{aligned}
x \%_{M M S E} & =\mathbf{W}_{M M S E} y=\left(\mathbf{H}^{H} \mathbf{H}+\sigma_{Z}^{2} \mathbf{I}\right)^{-1} \mathbf{H}^{H} \mathbf{Z} \\
& =x \%+\%_{M M S E}
\end{aligned}
$$

Where $2 /_{\text {MMSE }}=\left(\mathbf{H}^{H} \mathbf{H}+\sigma_{Z}^{2} \mathbf{I}\right)^{-1} \mathbf{H}^{H} \mathbf{Z}$ [7-9],[18].

\subsection{Ordered successive interference cancelation detection technique}

Generally, the linear detectors provide bad performance in comparison with nonlinear detection techniques. However, the linear detectors require a low hardware complexity in the course of the practical implementation. The performance of these linear detectors can be improved without increasing the hardware complexity using an ordered successive interference cancellation (OSIC) technique.

An ordered successive interference cancellation technique uses linear receiver's bank, each receiver detects only one of the parallel data sub-streams, and then the components of the detected signal will be canceled from the received signal at each stage successively. Finally, the remaining signal with least interference can be used in the next stage.

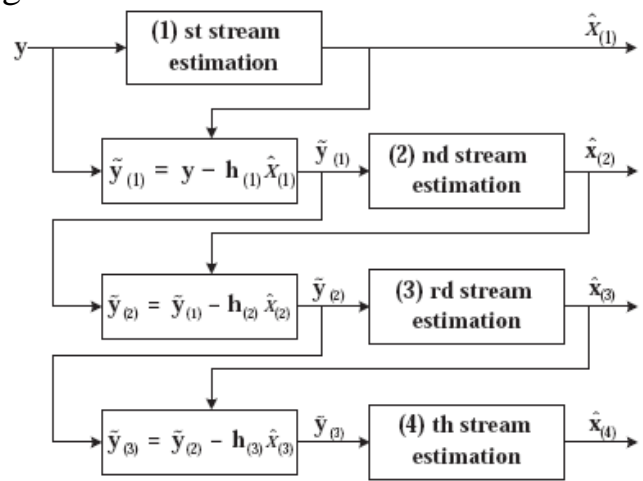

Fig. 3. OSIC detection approach for 4 spatial streams. 
Either ZF detector in equation (3) or MMSE detector in equation (5) can be used as symbols estimator in the consecutive OSIC stages [7-9], [18].

\section{Proposed robust fair-based SM-Mimo detector}

There are continuing efforts in order to develop detection techniques that hopefully achieving optimal or near-optimal performance with less complex computational processes. The proposed detector is based on the use of Fair M-Estimator. M-estimators are one of the most popular robust techniques which correspond to the ML (Maximum Likelihood) estimator [17].

Fair detector calculates the difference between the product of all transmitted signal vectors with the given channel matrix $\mathbf{H}$ and the observed signal vector at the receiving end, then calculates Fair function value at each difference, and hence determines the minimum Fair function value. Fair detector evaluates the estimate of the transmitted signal vector $\mathbf{x}$ using equations 8 and 9 [19].

$$
\begin{gathered}
\rho_{\text {Fair }}(\mathbf{y}-\mathbf{H x})=c^{2}\left(\frac{|\mathrm{y}-\mathbf{H} \mathbf{x}|}{c}-\log \left(1+\frac{|\mathrm{y}-\mathbf{H x}|}{c}\right)\right) \\
\mathrm{x}_{\text {Fair }}=\underset{\mathrm{x}}{\arg \min }\left(\rho_{\text {Fair }}\right)
\end{gathered}
$$

where $\rho_{\text {Fair }}($.$) is a symmetric, positive definite function with a unique minimum at 0$. The value $c$ is a tuning parameter that's used for trading-off high effectiveness with robustness. It was found that the tuning parameter $c$ has $95 \%$ efficiency at $1.3998[18,19]$. The $c=1.3998$ tuning parameter was used in the simulation.

\section{Simulation results}

The Monte-Carlo simulation results of the average BER will be partitioned into three subsections $A, B$, and $C$ as follow:

\subsection{Effect of Gaussian / non-Gaussian noises on the performance of examined detectors}

Table 1, illustrates the used simulation conditions, in this part.

Table 1.

The used parameters in simulation.

\begin{tabular}{|c|c|}
\hline Parameter & value \\
\hline \multirow{3}{*}{ Channel } & $\begin{array}{c}\text { Different noise channels } \\
\alpha=2,1, \text { and } 0.6\end{array}$ \\
\cline { 2 - 3 } & $\begin{array}{c}\text { Rayleigh fading channel over } \\
\text { independent transmit-receive links will } \\
\text { be used [8,9,18] }\end{array}$ \\
\hline MIMO conf. & $2 \times 2$ \\
\hline Modulation & QPSK \\
\hline \multirow{2}{*}{ Detectors } & ZF-OSIC [16], [17] \\
\cline { 2 - 2 } & MMSE-OSIC [16], [17] \\
\hline
\end{tabular}


Fig.4 - Fig.6 illustrate that when GGN (2) has distributions with heavy tails at $\alpha=1$, and $\alpha$ $=0.6$, the performance of the studied detectors become worse. Also, the MMSE-OSIC detector provides higher efficiency over the ZE-OSIC at all noise distributions $\alpha=2,1$, and 0.6.

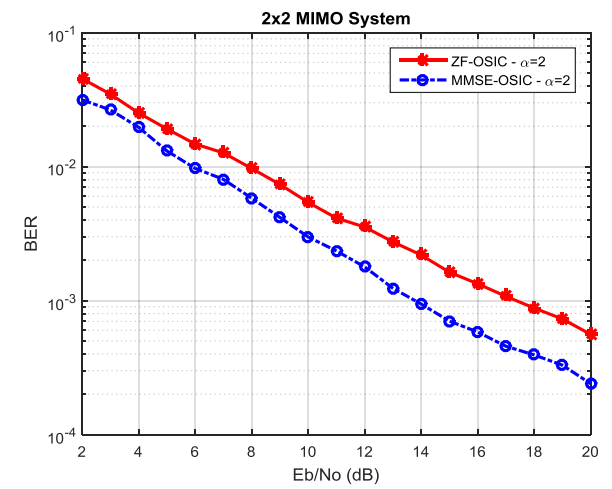

Fig. 4. Performance, of ZF- and MMSE- OSIC detectors, at $\alpha=2$, and SM-MIMO $2 \mathrm{X} 2$ system.

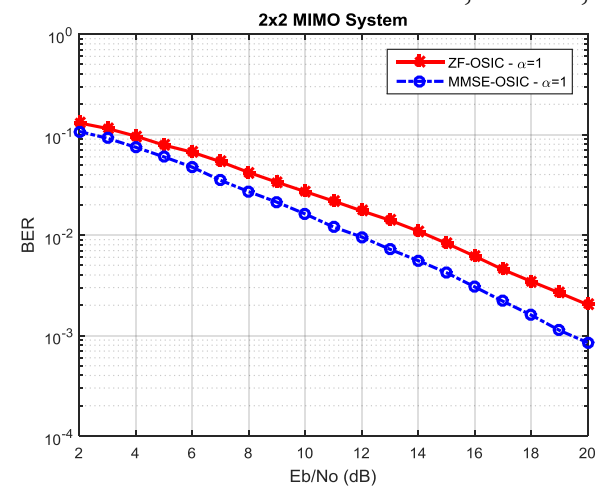

Fig. 5. Performance of ZF- and MMSE- OSIC detectors at $\alpha=1$, and SM-MIMO 2X2system.

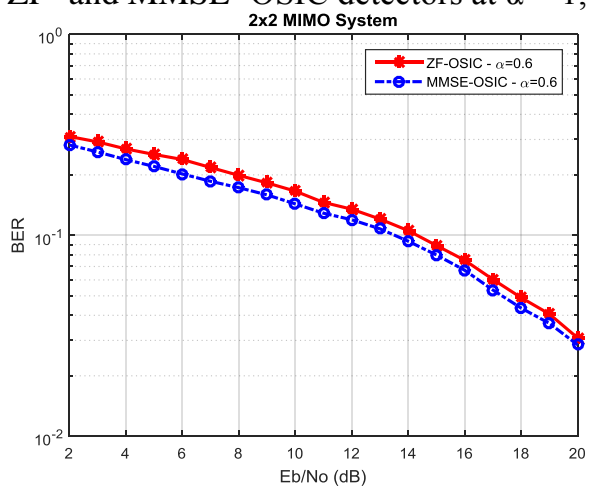

Fig. 6. Performance, of ZF- and MMSE- OSIC detectors, at $\alpha=0.6$, and SM-MIMO $2 \mathrm{X} 2$ system.

5.2. Effect of increasing the number of transmitting and receiving antennas on the performance of examined detectors in Gaussian / non-Gaussian noises

Table 2, illustrates the used simulation conditions, in this part. 
Table 2.

The used parameters in simulation.

\begin{tabular}{|c|c|}
\hline Parameter & value \\
\hline \multirow{3}{*}{ Channel } & $\begin{array}{c}\text { Different noise channels } \\
\alpha=2,1, \text { and } 0.6\end{array}$ \\
\cline { 2 - 2 } & $\begin{array}{c}\text { Rayleigh fading channel over independent } \\
\text { transmit-receive links will be used [8,9,18] }\end{array}$ \\
\hline MIMO conf. & $4 \times 4$ \& 8x8 \\
\hline Modulation & QPSK \\
\hline \multirow{2}{*}{ Detectors } & ZF-OSIC [16], [17] \\
\cline { 2 - 2 } & MMSE-OSIC [16], [17] \\
\hline
\end{tabular}

Fig.7 - Fig.12, show that as the number of transmitting and receiving antennas increase as the examined detectors performance goes better, at all noise distributions $\alpha=2,1$, and 0.6 .

It is clear that the increase in the number of the transmitting and receiving antennas in MIMO systems have a considerable role, in both increasing the transmission data rates through spatial multiplexing, and alleviating the bad impact of the existence of non-Gaussian noises, as presented in Fig.13. These noises are characterized by a heavy-tailed probability density functions.

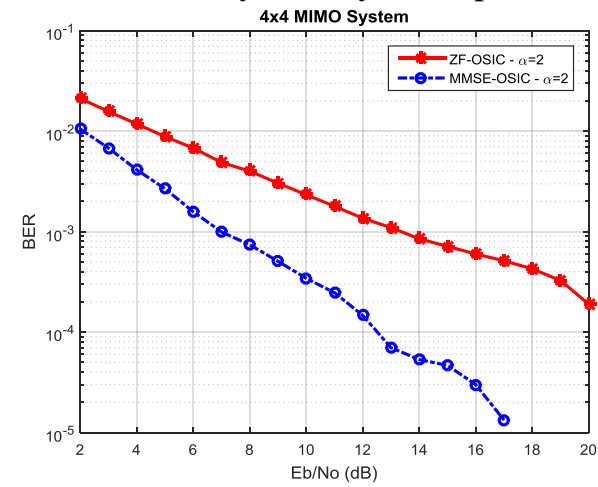

Fig. 7. Performance, of ZF- and MMSE- OSIC detectors, at $\alpha=2$, and SM-MIMO $4 \mathrm{X} 4$ system.

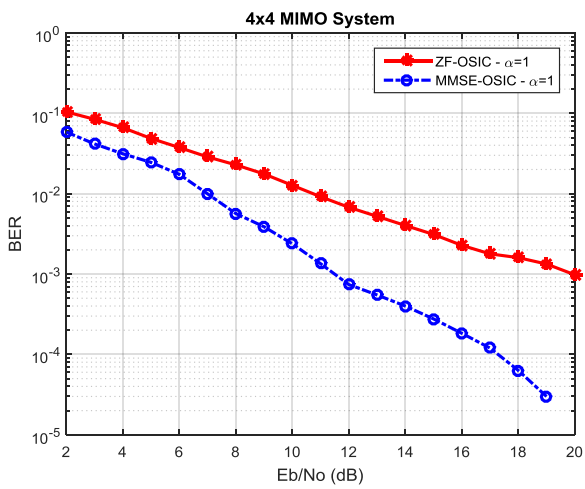

Fig. 8. Performance, for ZF- and MMSE- OSIC detectors, at $\alpha=1$, and SM-MIMO $4 \mathrm{X} 4$ system. 


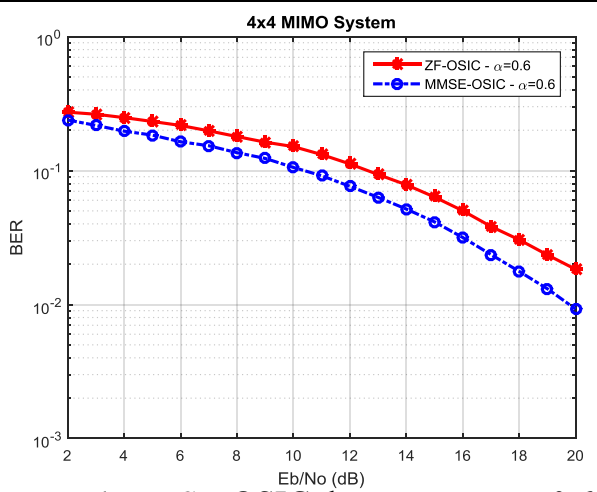

Fig. 9. Performance, of ZF- and MMSE-OSIC detectors, at $\alpha=0.6$, and SM-MIMO 4 X4system.

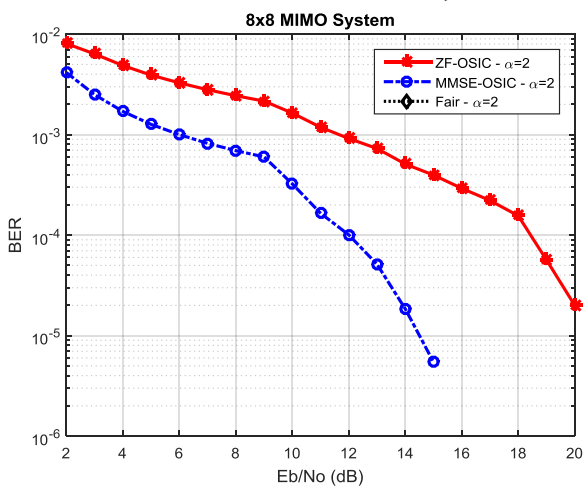

Fig. 10. Performance, of ZF- and MMSE- OSIC detectors, at $\alpha=2$, and SM-MIMO 8X8system.

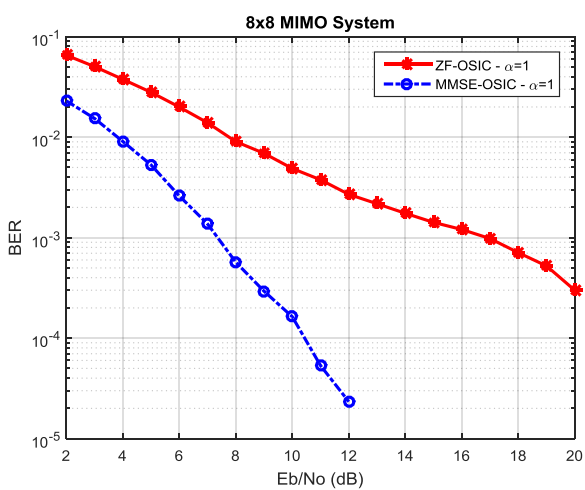

Fig. 11. Performance, of ZF- and MMSE- OSIC detectors, at $\alpha=1$, and SM-MIMO 8 X8 system.

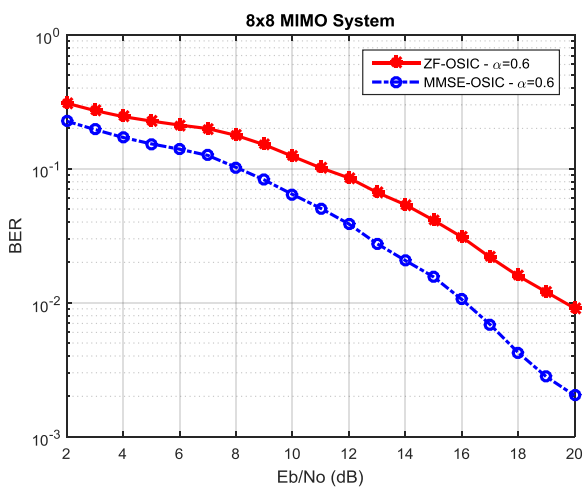

Fig. 12. Performance, of ZF-, and MMSE- OSIC detectors, at $\alpha=0.6$, and SM-MIMO $8 \mathrm{X} 8$ system. 


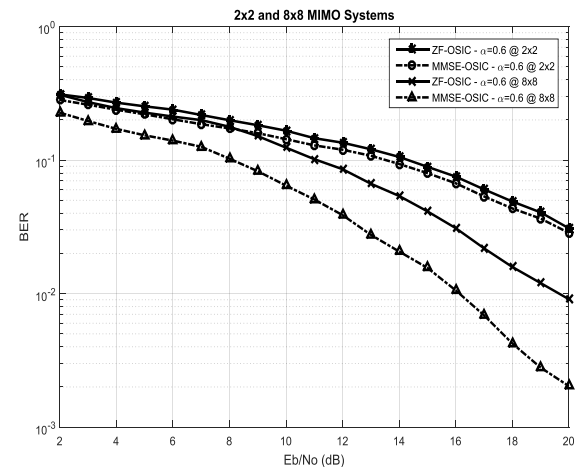

Fig. 13. BER versus SNR performance, for ZF-OSIC, and MMSE-OSIC detectors, at $\alpha=0.6$, and 2X2, and 8X8 SM-MIMO system.

5.3. The examination of the performance of the proposed Fair-detector in the presence of Gaussian / non-Gaussian noises

Table 3, illustrates the used simulation conditions, in this part.

Table 3.

The used parameters in simulation.

\begin{tabular}{|c|c|}
\hline Parameter & value \\
\hline \multirow{4}{*}{ Channel } & $\begin{array}{c}\text { Different noise channels } \\
\alpha=2,1, \text { and } 0.6\end{array}$ \\
\cline { 2 - 2 } & $\begin{array}{c}\text { Rayleigh fading channel over } \\
\text { independent transmit-receive links } \\
\text { will be used [8, 9, 18] }\end{array}$ \\
\hline MIMO conf. & $2 \times 2,4 \times 4 \& 8 \times 8$ \\
\hline Modulation & QPSK \\
\hline \multirow{3}{*}{ Detectors } & ZF-OSIC [16], [17] \\
\cline { 2 - 2 } & MMSE-OSIC [16], [17] \\
\cline { 2 - 2 } & Fair based-MIMO (Proposed) \\
\hline
\end{tabular}

Fig.14 - Fig.16, show that the proposed Fair detector outperforms ZE- and MMSEOSIC detectors at all noise distributions $\alpha=2,1$, and 0.6, and 2x2 MIMO configuration.

Fig.14 shows that the Fair detector has an obvious outperformance over the other two detectors at Gaussian noise distribution $\alpha=2$. Where Fig.15, and Fig.16, show the outperformance of the proposed detector in comparison with the examined detectors especially at higher SNRs; at noise distributions $\alpha=1$, and 0.6.

Fig. 17 - Fig. 21 show the tremendous impact of increasing the number of transmitting and receiving antennas on the overall performances of the examined detectors in general and the proposed Fair- detector specifically.

Fair detector successfully nullifies the Gaussian noise effect (at $\alpha=2$ ), and achieved $\mathrm{BER}=0$, for the full range of SNR values, at 8x8 MIMO configuration, while MMSE-OSIC start to nullify the Gaussian noise effect starting from SNR $=15 \mathrm{~dB}$, as shown in Fig. 10 . Fig. 20, depicts how the proposed detector provided $B E R=0$ (at $\alpha=1$ ), for SNR values starting from $\mathrm{SNR}=7 \mathrm{~dB}$, while MMSE-OSIC detector starts to nullify the noise effect starting from SNR = $12 \mathrm{~dB}$. Also, Fig. 21, depicted that Fair detector provided BER=0, at 
$\mathrm{SNR}=18 \mathrm{~dB}$ (at $\alpha=0.6$ ), while MMSE-OSIC detector didn't achieve BER=0 overall SNR examination values.

Fig.22 depicts the good impact of increasing the number of transmitting and receiving antennas on the performance of the proposed detector at $\alpha=0.6$.

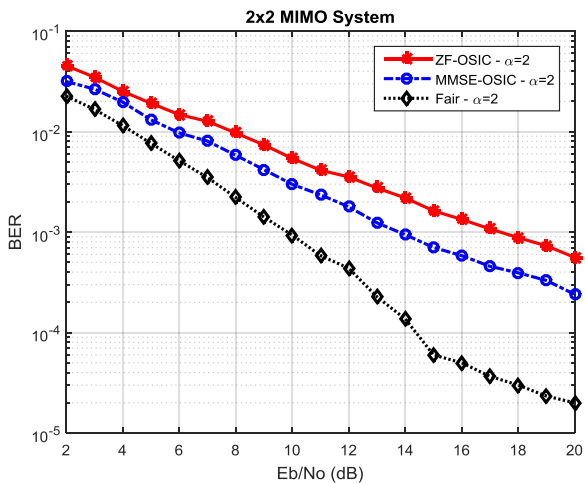

Fig. 14. Performance, of ZF-, MMSE- OSIC, and Fair detectors, at $\alpha=2$, and MIMO 2X2system.

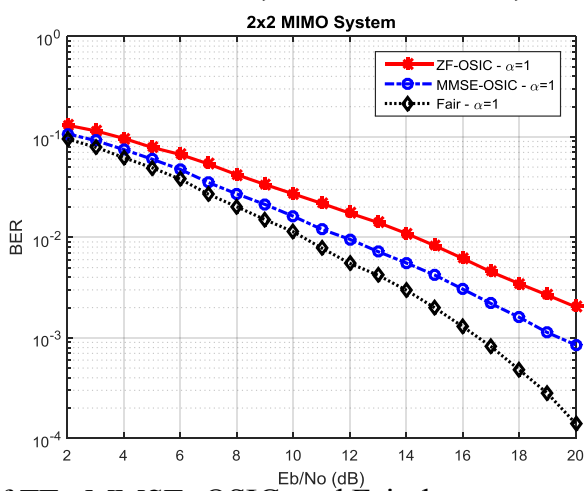

Fig. 15. Performance, of ZF-, MMSE- OSIC, and Fair detectors, at $\alpha=1$, and MIMO 2 X2system.

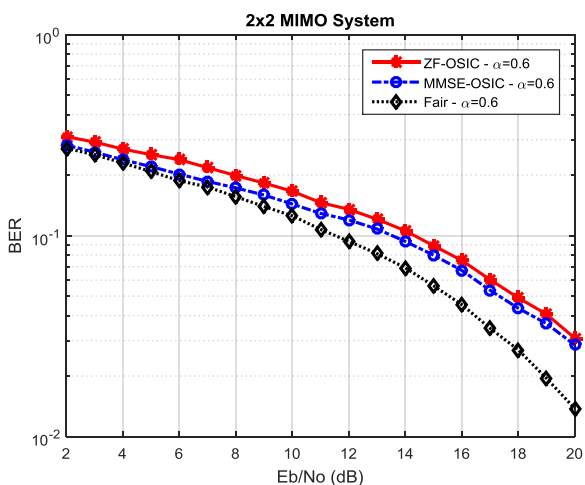

Fig. 16. Performance, of ZF-, MMSE- OSIC, and Fair detectors, at $\alpha=0.6$, and MIMO $2 \mathrm{X} 2$ system. 


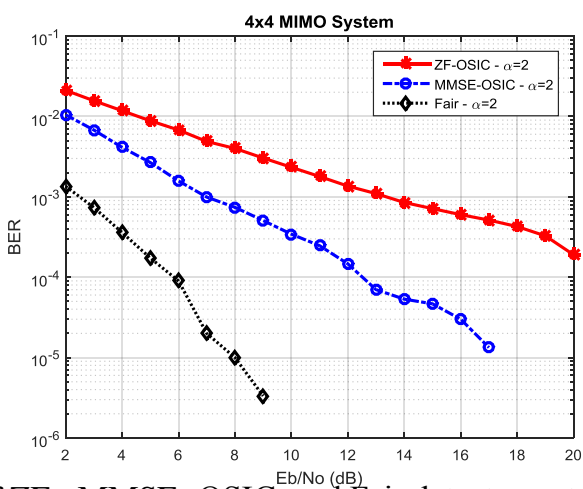

Fig. 17. Performance, of ZF-, MMSE- OSIC, and Fair detectors, at $\alpha=2$, and MIMO 4 X 4 system.

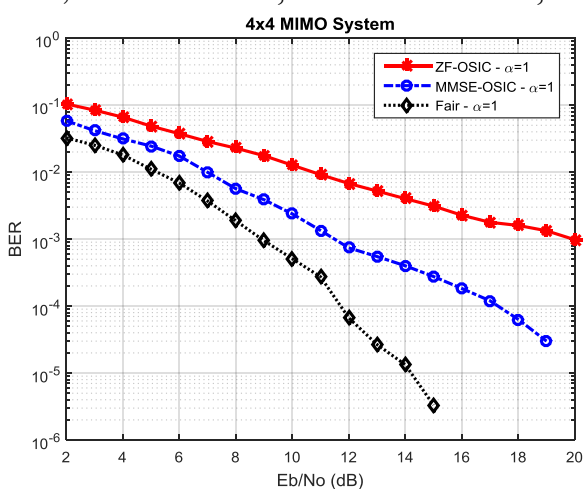

Fig. 18. Performance, of ZF-, MMSE- OSIC, and Fair detectors, at $\alpha=1$, and MIMO 4 X4 system.

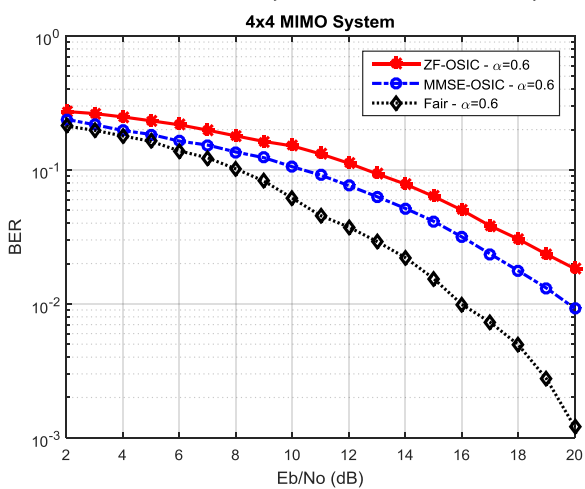

Fig. 19. Performance, of ZF-, MMSE- OSIC, and Fair detectors, at $\alpha=0.6$, and MIMO 4 X4system.

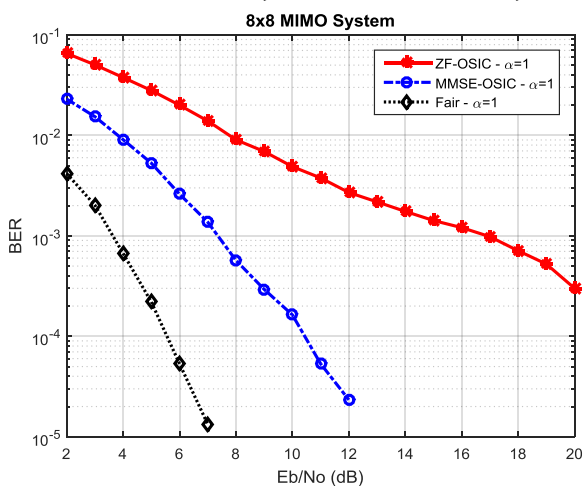

Fig. 20. BER versus SNR performance, forZF-OSIC, MMSE-OSIC,and Fair detectors at $\alpha=1$, and 8X8MIMO system. 


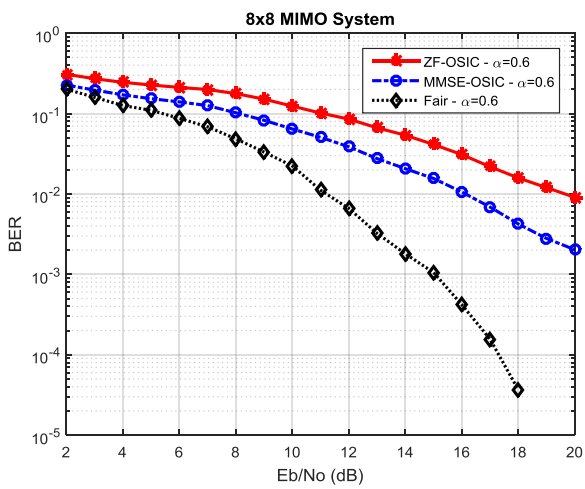

Fig. 21. BER versus SNR performance, for ZF-OSIC, MMSE-OSIC, and Fair detectors, at $\alpha=$ 0.6 , and 8 X8 MIMO system.

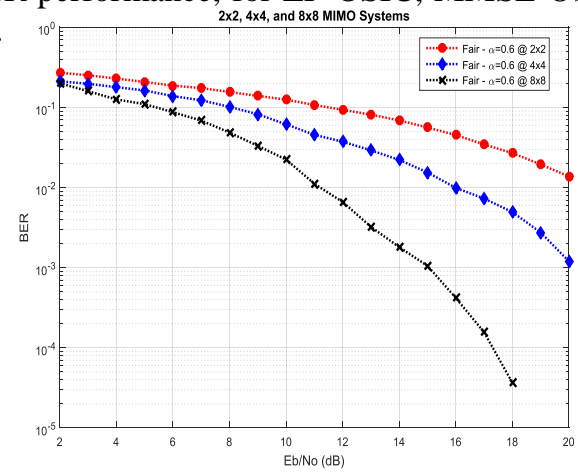

Fig. 22. BER versus SNR performance, for ZF-OSIC, MMSE-OSIC, and Fair detectors, at $\alpha=$ 0.6 , and $2 \times 2,4 \times 4$ and 8 X8 SM-MIMO system.

\subsection{Computational complexity of the examined detectors}

The efficient practical implementation of the MIMO detectors is the main challenge in MIMO wireless systems. Several detectors offering different trade-offs between good performance and computational complexity have been discussed in literature [20]. In this subsection, the examined detectors will be evaluated in terms of computational complexity criteria. The computational complexity of the detectors will only be computed in terms of FLOPS (Floating Point Operations per Second (additions, multiplications etc.)).

The computational complexity of the examined detectors in terms of the number of transmitting antennas $N_{t}$, number of receiving antennas $N_{r}$ and constellation size $M$ is tabulated in Table 4 [20].

Table 4.

Comparison of the MIMO detectors.

\begin{tabular}{|c|c|}
\hline Detector & Complexity (FLOPS) \\
\hline ZF-OSIC & $N_{t}^{4}+\frac{5}{3} N_{t}^{3}+\frac{8}{3} N_{t}^{3} N_{r}+\frac{3}{4} N_{t}^{2}+\frac{7}{2} N_{t}^{2} N_{r}+\frac{55}{6} N_{t} N_{r}-\frac{17}{12} N_{t}+\frac{1}{2} N_{t} \log _{2}(M)$ \\
\hline MMSE-OSIC & $N_{t}^{4}+\frac{5}{3} N_{t}^{3}+\frac{7}{3} N_{t}^{3} N_{r}+N_{t}^{2}+\frac{7}{2} N_{t}^{2} N_{r}+\frac{7}{6} N_{t} N_{r}-\frac{1}{6} N_{t}+\frac{1}{2} N_{t} \log _{2}(M)$ \\
\hline Fair & $M^{N_{t}}$ \\
\hline
\end{tabular}


JES, Assiut University, Faculty of Engineering, Vol. 46, No. 3, May 2018, pp.317-332

The generalized expressions given in Table 4 are validated in Table 5 for a $2 \times 2,4 \times 4$, and 8x8 MIMO systems employing QPSK, 16-QAM, and 64-QAM modulation schemes with various detectors at the receiver.

Table 5.

Complexity analyses for QPSK, 16QAM, 64QAM modulation schemes, and 2x2, 4x4, and 8x8 MIMO systems.

\begin{tabular}{|c|c|c|c|c|c|c|c|c|c|}
\hline \multirow{3}{*}{ Detector } & \multicolumn{9}{|c|}{ Complexity (FLOPS) } \\
\hline & \multicolumn{3}{|c|}{ QPSK } & \multicolumn{3}{|c|}{ 16-QAM } & \multicolumn{3}{|c|}{ 64-QAM } \\
\hline & $2 \times 2$ & $4 \times 4$ & $8 \times 8$ & $2 \times 2$ & $4 \times 4$ & $8 \times 8$ & $2 \times 2$ & $4 \times 4$ & $8 \times 8$ \\
\hline $\begin{array}{c}\text { ZF- } \\
\text { OSIC }\end{array}$ & 138 & 1426 & 18295 & 141 & 1431 & 18303 & 143 & 1434 & 18311 \\
\hline $\begin{array}{c}\text { MMSE- } \\
\text { OSIC }\end{array}$ & 105 & 1222 & 16444 & 107 & 1226 & 16452 & 109 & 1230 & 16460 \\
\hline Fair & 16 & 256 & 65536 & 256 & 65536 & $\begin{array}{c}4.2950 \\
\mathrm{e}+09\end{array}$ & 4096 & 16777216 & $\begin{array}{c}2.8147 \\
\mathrm{e}+14 \\
\end{array}$ \\
\hline
\end{tabular}

It is clear from Table 4 that the computational complexity of the examined detectors depends on the number of transmitting and receiving antennas, and the constellation size of the modulation schemes. Also, it is clear that Fair detector has an exponential complexity in the number of transmit antennas and constellation size.

In general, Table 5 shows that Fair detector exhibits higher computational complexity than ZF-OSIC, and MMSE-OSIC detectors.

From the perspective of hardware and due to the tremendous advances in the manufacturing of microprocessors these computational complexities are considered as reasonable computational complexities. For example a single intel Xeon E5-2600v3 processor is capable of more than half a TeraFLOPS (500 GFLOPS) [21].

\section{Conclusion}

The Fair-based SM-MIMO detector is proposed in this work. The proposed detector outperforms the conventional linear MIMO detectors, including the ZF- and MMSE- OSIC detectors, at all noise distributions. The proposed detector achieves a noticeable performance improvement where it successfully nullifies the Gaussian noise effect (at $\alpha=$ 2 ), and achieves $B E R=0$, for the full range of SNR values, at 8x8 MIMO configuration. The proposed detector designed for LTE and LTE-advanced wireless communication systems. As a result of the tremendous advance in the manufacturing of microprocessors the practical implementation of the proposed detector becomes feasible.

\section{REFERENCES}

[1] I. E. Telatar, Capacity of multi-antenna Gaussian channel", European Trans. Telecomm., vol. 10, no. 6, pp. 585-595, Nov. Dec. 1999.

[2] G. Foschini, and M. Gans, On limits of wireless communications in a fading environment when using multiple antennas", Wireless Personal Commun., vol. 6, no. 3, pp. 311-335, Mar. 1998.

[3] Syed A., Aamir H., and Qamar. Optimal Decoders for 4G LTE Communication, Lecture Notes on Information Theory Vol. 1, No. 4, December 2013.

[4] V. A. Bogdanovich and A. G. Vostretsov. "Application of the Invariance and Robustness Principles in the Development of Detection Algorithms for Wideband Communications 
Mohamed H. Essai, fair-M-estimator based mimo detector and mimo system investigation ....

Systems," Journal of Communications Technology and Electronics, vol. 54, no. 11, pp. 1283-1291, Nov. 2009, (C) Pleiades Publishing, Inc., 2009. original Russian text (CV.A. Bogdanovich, A.G. Vostretsov, 2009, published in Radiotekhnika i Elektronika, vol. 54, no. 11, pp. 1353-1362, 2009.

[5] A. G. Vostretsov, V. A. Bogdanovich, Mohamed H. Essai "Robust Detection Algorithm For Future 4G wireless Communication Systems", XIV international conference of young specialists on Micro/Nanotechnologies and electronics devices, 1-5 July 2013.

[6] V.A. Bogdanovich, A.G. Vostretsov, M. H. Essai CDMA Robust Demodulation Algorithm in The Presence of Multiple Access Interference. Proceedings. The 5th International Forum on Strategic Technology., Korea. - Ulsan, P. 100 - 104, Oct. 13-15, 2010.

[7] Y. S. Cho, J. Kim, W. Y. Yang, and C. G. Kang, MIMO-OFDM Wireless Communications with Matlab, John Wiley \& Sons (Asia) Pte Ltd, 2010.

[8] M. H. Essai, "MIMO-aided robust LTE detectors in actual noise environments," 2014 10th International Computer Engineering Conference (ICENCO), Giza, 2014, pp. 121127.doi:10.1109/ICENCO.2014.7050443. http://ieeexplore.ieee.org/document/7050443/ . Last access date 01/03/2018.

[9] M. H. Essai, "SM-MIMO effect on the performance of LTE detectors in uncertain noise environments," 2014 12th International Conference on Actual Problems of Electronics Instrument Engineering (APEIE), Novosibirsk, 2014, pp.352-356. doi:10.1109/APEIE.2014.7040916. http://ieeexplore.ieee.org/document/7040916/. Last access date 01/03/2018.

[10] D. Middleton. Statistical-physical models of electromagnetic interference. IEEE Transactions on Electromagnetic Compatibility, EMC-19(3): 106-27, August 1977.

[11] D. Middleton. Non-Gaussian Noise Models in Signal Processing for Telecommunications: New Methods and Results for Class A and Class B Noise Models. IEEE Transactions on Information Theory, 45(4): 1129-49, 1999.

[12] Michael L. Honig, advances in multiuser detection, Northwestern University, John Wiley \& Sons, 2009.

[13] N. Jindal, J. G. Andrews, and S. P. Weber, "Rethinking mimo for wireless networks: Linear throughput increases with multiple receive antennas," in Proc. IEEE Int. Conf. on Commun, Dresden, Germany, June 2009, pp. 1-5.

[14] Nihar. Multi-antenna communication in ad hoc networks: Achieving mimo gains with simo transmission. IEEE Trans. Commun. [Online]. Available: http://arxiv.org/pdf/0809.5008v2 . Last access date 07/03/2018.

[15] O. B. S. Ali, C. Cardinal, and F. Gagnon. "Performance of optimum combining in a poisson field of interferers and rayleigh fading channels". IEEE Trans. Commun. [Online]. Available: http://arxiv.org/pdf/1001.1482v3. Last access date 07/03/2018.

[16] G. J. Foschini, "Layered space-time architecture for wireless communication in a fading environment when using multiple antennas," The Bell Sys. Tech. Journal, No. 1, pp. 41-59, 1996.

[17] P. W. Wolniansky, G. J. Foschini, G. D. Golden, R. A. Valenzuela, "V-BLAST: An Architecture for realizing very high data rates over the rich scattering wireless channel," 1998 URSI International Symposium on Signals, Systems, and Electronics, , pp. 295-300, 29 Sep.-2 Oct. 1998.

[18] Mohammed H. Essai, "Huber M-estimator based LTE robust detector", 11th International Forum on Strategic Technology (IFOST), Novosibirsk, pp. 281-285, 2016. doi: 10.1109/IFOST.2016.7884106. http://ieexplore.ieee.org/document/7884106/. Last access date $01 / 03 / 2018$

[19] Huber, P.J.: Robust Statistics. John Wiley and Sons, New York (1981).

[20] Auda M.Elshokry, "Complexity and performance evaluation of detection schemes for spatial multiplexing MIMO systems", M.sc thesis, Islamic university, Jan 2010.

[21] White Paper "Optimizing Performance with Intel ®Advanced Vector Extensions", September 2014.

Available:https://computing.llnl.gov/tutorials/linux_clusters/intelAVXperformanceWhitePaper.p df. Last access date 06/03/2018. 


\section{كاثف نظام الإتصالات متعدد المدخلات و متعدد المخرجات المعتمد

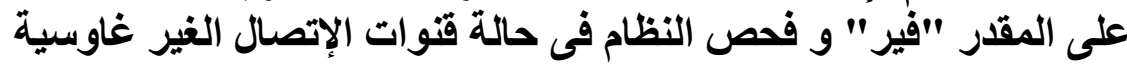

$$
\text { الملخص العربي }
$$

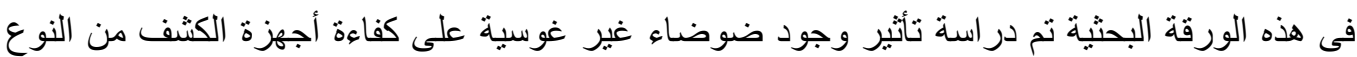
و النوع (OSIC-MMSE)

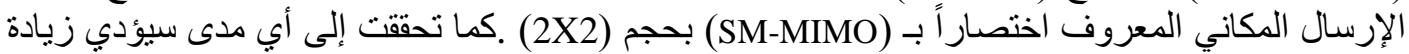

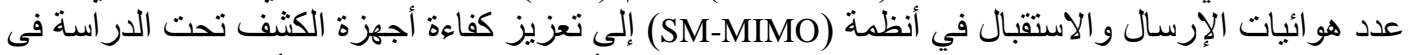

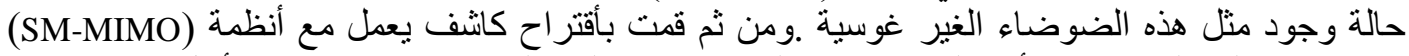

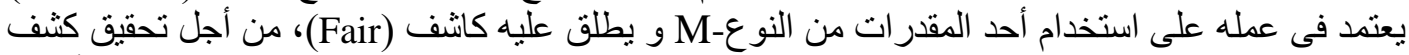

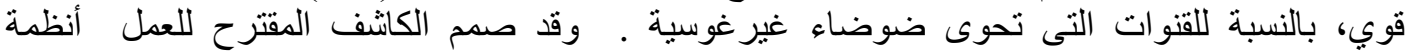

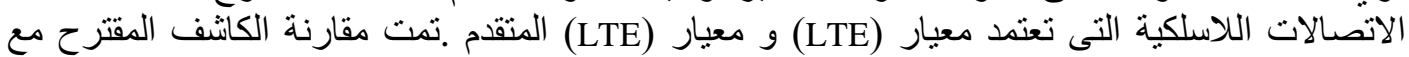
أجهزة الكثف المذكورة آنفا من حيث معدل الخطأ في البتات و التعقيد الحسابى .نتائج المحاكاة تظهر الأداء الجو هري للكاشف المقترح مقارنة مع أجهزة الكثف التي تم درد در استها. 